In der ersten und in der letzten Summe in (35) verschwinden hierbei alle Summanden, bei denen $\mu \neq v$ ist. In der mittleren Summe sind diejenigen Summan- den von Null verschieden, für die $\mu=m+v$ oder $\mu=m-v$ ist. Mit der Abkürzung $q=\frac{\hbar^{2}}{2 m_{0}} \frac{4 \pi^{2}}{L^{2}}$ ergeben sich die folgenden Rekursionsformeln

$$
\begin{array}{ll}
\text { für } \mu=0: \quad-C_{0 k} \cdot E_{k}+\frac{V_{0}}{2} C_{m k}=0, & \left(q \mu^{2}-E_{k}\right) C_{\mu k}+\frac{V_{0}}{2}\left(C_{m-\mu, k}+C_{m+\mu, k}\right)=0, \\
\text { für } \mu<m: \quad\left(q^{2} m^{2}-E_{k}\right) C_{m k}+\frac{V_{0}}{2}\left(2 C_{0 k}+C_{2 m, k}\right)=0, \\
\text { für } \mu=m: \\
\text { für } \mu>m: \quad\left(q^{2} \mu^{2}-E_{k}\right) C_{\mu k}+\frac{V_{0}}{2}\left(C_{\mu-m, k}+C_{\mu+m, k}\right)=0 .
\end{array}
$$

Bei Beträgen von $V_{0}<10 \mathrm{eV}$ konvergiert die Folge der betrachteten Koeffizienten sehr schnell. Setzt man von einem genügend großen Wert $M$ des Index $\mu$ an alle folgenden Koeffizienten $C_{\mu k}=0$, so ergibt sich ein System von Eigenwertgleichungen. Die Eigenwerte $E_{k}$ und die zugehörigen Eigenvektoren $C_{u k}$ können leicht mit Hilfe einer elektronischen Rechenanlage berechnet werden. Die Ermittlung der Koeffizienten $S_{u k}$ erfolgt in analoger Weise. Tab. 4 zeigt das Ergebnis für die Fälle $m=2 ; 3 ; 8 ; 9$ und $V_{0}=2,4 \mathrm{eV}$.

\title{
Lumineszenzuntersuchungen am kristallinen Phenanthren
}

\section{A. Tschampa}

I. Physikalisches Institut der Universität Gießen

(Z. Naturforschg. 22 a, 112-118 [1967] ; eingegangen am 12. Oktober 1966)

\begin{abstract}
Despite extreme purification the luminescence of polycrystalline phenanthrene is strongly influenced by remaining impurities. Besides fluorescence the samples show a delayed fluorescence and phosphorescence and the normal triplet phosphorescence of an unknown impurity. After intense excitation with uv light both components of the delayed luminescence can be observed for several minutes. Thermal stimulation of both fluorescence and phosphorescence is possible. To describe the observed luminescence behavior an energy level diagram is proposed with exciton traps below the singlet and triplet band of the crystal. The traps are due to crystal defects and photochemical products respectively. A part of the delayed fluorescence is based on triplet triplet annihilation and delayed phosphorescence on thermal release of trapped excitons.
\end{abstract}

Das Fluoreszenzspektrum des kristallinen Phenanthrens beginnt mit dem 0,0-Übergang bei 28600 $\mathrm{cm}^{-1}{ }^{1-3}$. In Lösungen von Alkohol, Pentan u. a. verschiebt es sich nach etwa $28900 \mathrm{~cm}^{-14^{-6}}$. In solchen Lösungen wird bei tieferen Temperaturen eine $\mathrm{T} \rightarrow \mathrm{S}$-Phosphoreszenz beobachtet mit einem 0,0 Übergang bei $21700 \mathrm{~cm}^{-15^{-8}}$. Das Kristallumineszenzspektrum enthält in der Regel noch beträchtliche Emissionsanteile von Verunreinigungen, darunter besonders Anthracen.

1 D. S. McClure, J. Chem. Phys. 25, 481 [1956].

2 H. C. Wolf, Z. Naturforschg. 13 a, 420 [1958].

${ }^{3}$ K.W.Benz u. H.C.Wolf, Z. Naturforschg. 19 a, 181 [1964].

4 M. Nakamizo u. Y. Kanda, Spectrochim. Acta 19, 1235 [1963].

5 T. Azumi u. S. P. McGlynn, J. Chem. Phys. 37, 2413 [1962].

${ }_{6}^{6}$ L. Pesteil u. M. Rabaud, J. Chim. Phys. 59, 167 [1962].

7 H. Gropper u. F. DörR, Ber. Bunsenges. 67, 46 [1963].

8 H. Sponer, in: Kallmanx u. Spruch, Luminescence of Organic and Inorganic Materials, John Wiley \& Sons, Inc., New York-London 1962, S. 143.
Wie bei einigen anderen Kohlenwasserstoffen tritt auch beim Phenanthren im Dampfzustand ${ }^{9,10}$, in flüssigen ${ }^{11,12}$ und festen Lösungen ${ }^{13}$ und im kristallinen Zustand ${ }^{14-16}$ eine verzögerte Fluoreszenz auf. Über die Energiespeicherungs- und Rücklieferungsprozesse gibt es verschiedene Vorstellungen. SPONER und Mitarbeiter ${ }^{14}$, die wie Blake und McClure ${ }^{15}$ das Phänomen an Naphthalin- und Phenanthrenkristallen untersuchten, machten dafür den Einfang von Elektronen in Haftstellen verantwortlich. An-

9 R. Williams, J. Chem. Phys. 28, 577 [1958].

10 B. Stevens, E. Hutton u. G. Porter, Nature 185, 917 [1960].

11 C. A. Parker u. C. G. Hatchard, Proc. Chem. Soc. 1962 , 147.

12 C. A. Parker u. C. G. Hatchard, Trans. Faraday Soc. 59, 284, [1963].

13 T. Azumi u. S. P. McGlynx, J. Chem. Phys. 38, 2773; 39, 1186,3533 [1963].

14 H. Sponer, Y. Kanda u. L. A. Blackwell, J. Chem. Phys. 29, 721 [1958]. 
dere Autoren ${ }^{13,16-18}$ sehen in dem Zusammenwirken zweier Triplettzustände (für Kristall und feste Lösungen) oder in der Bildung längerlebiger angeregter Dimere (für flüssige Lösungen und Dampfzustand) ${ }^{9-12}$ den Energielieferanten. Zu dieser Frage bringt die vorliegende Arbeit einige neue Beobachtungen und Erklärungsversuche.

\section{Die Präparation der Proben}

Phenanthren "white label grade" der Firma East. man Organic Chemicals wurde nach einem von $\mathrm{C}_{\mathrm{LAR}}{ }^{19}$ angegebenen Verfahren weitgehend von Anthracen (bis auf etwa $10^{-6}$ Molanteile) befreit. Anschließend wurde eine weitere Reinigung durch Zonenschmelzen in einem $50 \mathrm{~cm}$ langen vertikal aufgehängten Rohr versucht. Das Rohr wurde nach dem Füllen unter Atmosphärendruck abgeschlossen. Nach Passieren von 30 oder 50 Zonen wurde das Material des mittleren Rohrteiles umgefüllt und das Verfahren wiederholt. Proben, die in dieser Weise 30, 50 oder 100 Zonen durchlaufen hatten, werden mit $\mathrm{P}_{30}, \mathrm{P}_{50}, \mathrm{P}_{100}$ bezeichnet $\left(\mathrm{vgl} .{ }^{3}\right)$. Es wurde jedoch keine vollständige Beseitigung des Anthracens erreicht (siehe Abb. 1).

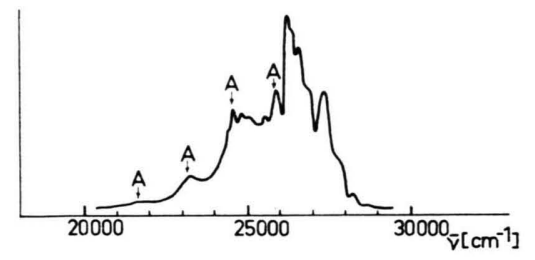

Abb. 1. Fluoreszenz von Phenanthren $\mathrm{P}_{50}$ bei $77^{\circ} \mathrm{K}$. $\mathrm{A}=$ Anthracenbanden.

Daher wurde versucht, während des Zonenreinigungsprozesses das Anthracen innerhalb der Schmelzzonen photochemisch zu zersetzen. Hierzu wurde das Licht einer Xenonhochdrucklampe mit einer Quarzlinse auf eine geschmolzene Zone abgebildet. Durch die Bestrahlung wurde das Material zunächst gelb, später braun verfärbt. Die so gebildeten Umwandlungsprodukte sammelten sich nach Durchlaufen genügend vieler Zonen am unteren Rohrende, und der Verfärbungsprozeß nahm ab, da der im Rohr vorhandene Sauerstoff aufgebraucht wurde. Proben, die in dieser Weise $n$ Zonen unter Bestrahlung durchlaufen haben, werden durch einen zusätzlichen oberen Index $n$ gekennzeichnet: $\mathrm{P}_{m}^{n}$. Abb. 2 zeigt das Fluoreszenzspektrum einer Probe $\mathrm{P}_{450}^{25}$ bei verschiedenen Temperaturen. Die charakteristischen Anthracenmaxima sind darin nicht mehr zu erkennen; der Emissionsschwerpunkt ist nach höheren Wellenzah-

15 N. W. Blake u. D. S. McClure, J. Chem. Phys. 29, 722 [1958].

16 M. Z ZaNDER, Ber. Bunsenges. 68, 301 [1964].

17 H. Sternlicht, G. C. Nieman u. G. W. Robinson, J. Chem. Phys. 38, 1326 [1963]. len verschoben. Das spricht für einen höheren Reinheitsgrad. Die starke Veränderung der Emissionsstruktur mit der Temperatur deutet aber immer noch auf Beteiligung von Verunreinigungen oder Gitterstörstellen an der Fluoreszenzemission (siehe z. B. auch BENz und $\left.\mathrm{WoLF}^{3}\right)$.

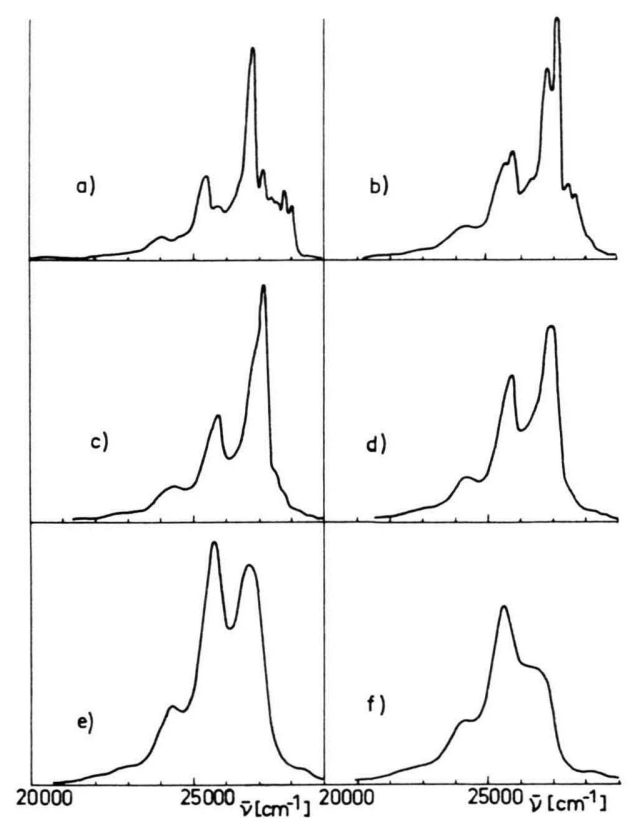

Abb. 2. Fluoreszenz von Phenanthren $\mathrm{P}_{450}^{25}$ bei a) 77 , b) 130 , c) 160 , d) 225 , e) 295 , f) $350^{\circ} \mathrm{K}$.

\section{Die apparative Anordnung}

Zur Aufnahme der Spektren und Untersuchung der Abklingvorgänge wurden die polykristallinen Proben mit der Strahlung einer Xenonhochdrucklampe angeregt. Für die Untersuchung der stationären Fluoreszenz wurde durch eine Filterkombination der Bereich 2200 bis $3400 \AA$ zur Anregung ausgewählt, während bei Untersuchung des Nachleuchtens in der Regel die Gesamtstrahlung zur Anregung verwandt wurde.

Die Proben konnten in einer zylindrischen Vertiefung eines Kupferblocks gekühlt und mit einer Heizvorrichtung mit wählbarem Temperaturgradienten, in der Regel $80^{\circ} \mathrm{K} / \mathrm{min}$, aufgeheizt werden. Die Temperatur wurde am Boden der Probenküvette gemessen. Die Strahlengänge für das anregende Licht und die zu untersuchende Emissionsstrahlung konnte wahlweise durch zwei Photoverschlüsse oder durch einen rotierenden Zylinder mit zwei um $180^{\circ}$ gegeneinander versetzten Öffnungen (abgewandeltes BEcqered-Phosphoroskop) periodisch unterbrochen werden.

18 R. G. Kepler, J. C. Caris, P. Avakian u. E. Abramson, Phys. Rev. Letters 10, 400 [1963].

19 E. Clar, Ber. Dtsch. Chem. Ges. 65, 852 [1963] ; - Aromatische Kohlenwasserstoffe, Polycyclische Systeme, Springer, Berlin-Göttingen-Heidelberg 1952. 
Je nach der Art des Experimentes wurde die Lumineszenzstrahlung direkt oder nach Durchgang durch einen Monochromator (B e c k m a n DU Quarzspektrometer) mit einem Photomultiplier (R C A 7265) gemessen und oszillographisch oder mit einem Schreiber registriert.

\section{Beobachtungsergebnisse}

a) Die stationäre Lumineszenz

Das Emissionsspektrum der gereinigten Phenanthrenproben zeigt zwei deutlich getrennte Emissionsgebiete: eine kurzwellige Emission im Gebiet $28600 \mathrm{~cm}^{-1}-20000 \mathrm{~cm}^{-1}$ mit praktisch temperaturunabhängiger Intensität im Bereich $77^{\circ} \mathrm{K}$ bis

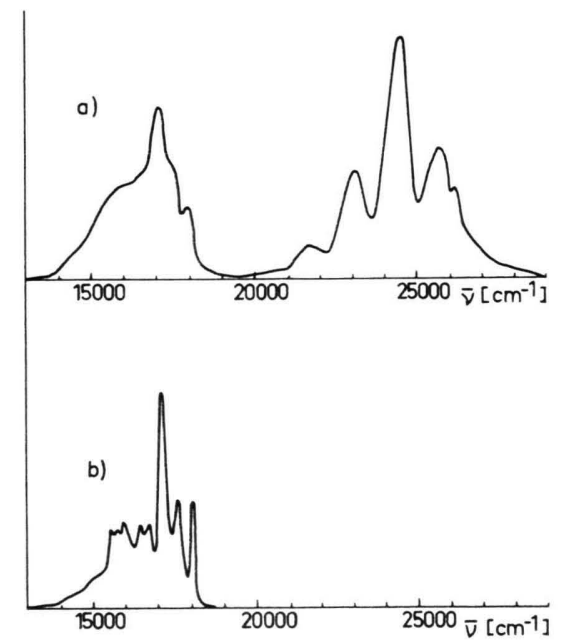

Abb. 3. Phenanthren-Nachleuchten (Probe $\mathrm{P}_{30}$ ) a) bei 240 und b) bei $77^{\circ} \mathrm{K}$.

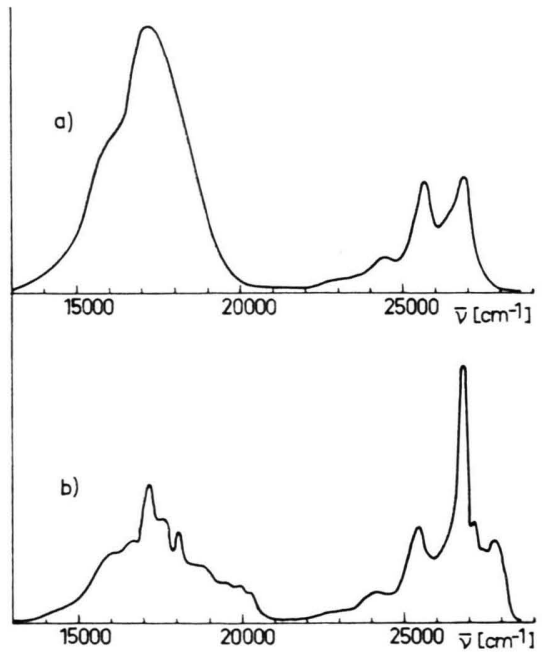

Abb. 4. Phenanthren-Nachleuchten (Probe $\mathrm{P}_{450}^{25}$ ) a) bei 240 und b) bei $77^{\circ} \mathrm{K}$.
Zimmertemperatur und eine längerwellige Emission im Gebiet $18000-14000 \mathrm{~cm}^{-1}$, deren relativer Anteil bei Zimmertemperatur nur $1-10 \%$ beträgt, die aber mit abnehmender Temperatur stark ansteigt.

Die Struktur beider Emissionsgebiete hängt von den benutzten Reinigungsoperationen ab. In den nur zonengereinigten Proben sind in der kurzwelligen Gruppe Anthracenbanden nachweisbar, die in den photochemisch behandelten Proben fehlen. Aber auch in den letzteren gehören wahrscheinlich nicht alle Banden dem Phenanthren an, sondern teilweise unbekannten fluoreszierenden Zentren (vgl. ${ }^{3}$ ).

\section{b) Das Nachleuchten}

Die Proben zeigen ein Nachleuchten, das bis zu Zeitdauern der Größenordnung 10 min verfolgt werden kann und dessen Spektrum mit den oben genannten beiden Emissionsgebieten identisch ist (Abb. $3,4)$. Jedoch unterscheiden sich die beiden Komponenten in der Temperaturabhängigkeit sehr deutlich voneinander (Abb. 5).

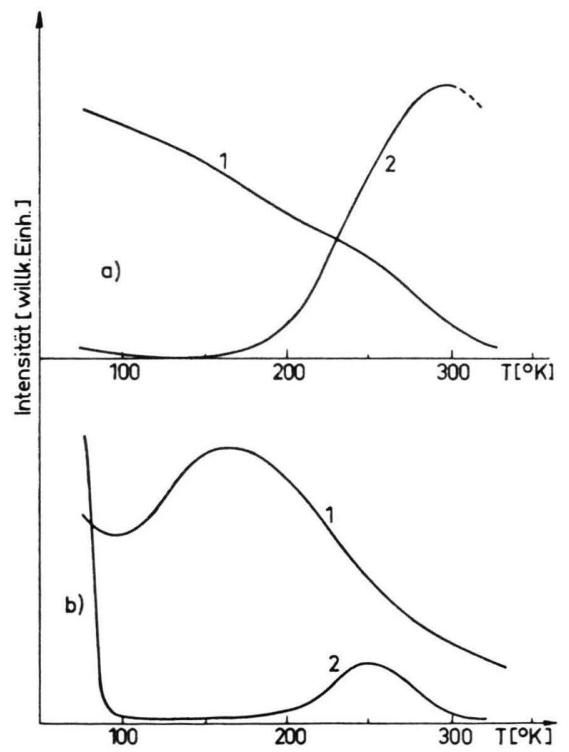

Abb. 5. Temperaturabhängigkeit von stationärer Phosphoreszenz (1) und verzögerter Fluoreszenz (2) der Proben a) $P_{30}$ und b) $\mathrm{P}_{450}^{25}$. Die Intensitäten von (1) und (2) sind nicht vergleichbar.

Auf Grund der spektralen Identität mit der stationären Fluoreszenz ist die kurzwellige Emission des Nachleuchtens als verzögerte Fluoreszenz anzusprechen. Die langwellige Komponente dürfte einem $\mathrm{T} \rightarrow \mathrm{S}$-Übergang zuzuordnen sein. Nach SPoner ${ }^{8}$ liegt die 0,0-Bande der $\mathrm{T} \rightarrow \mathrm{S}$-Phosphoreszenz von 

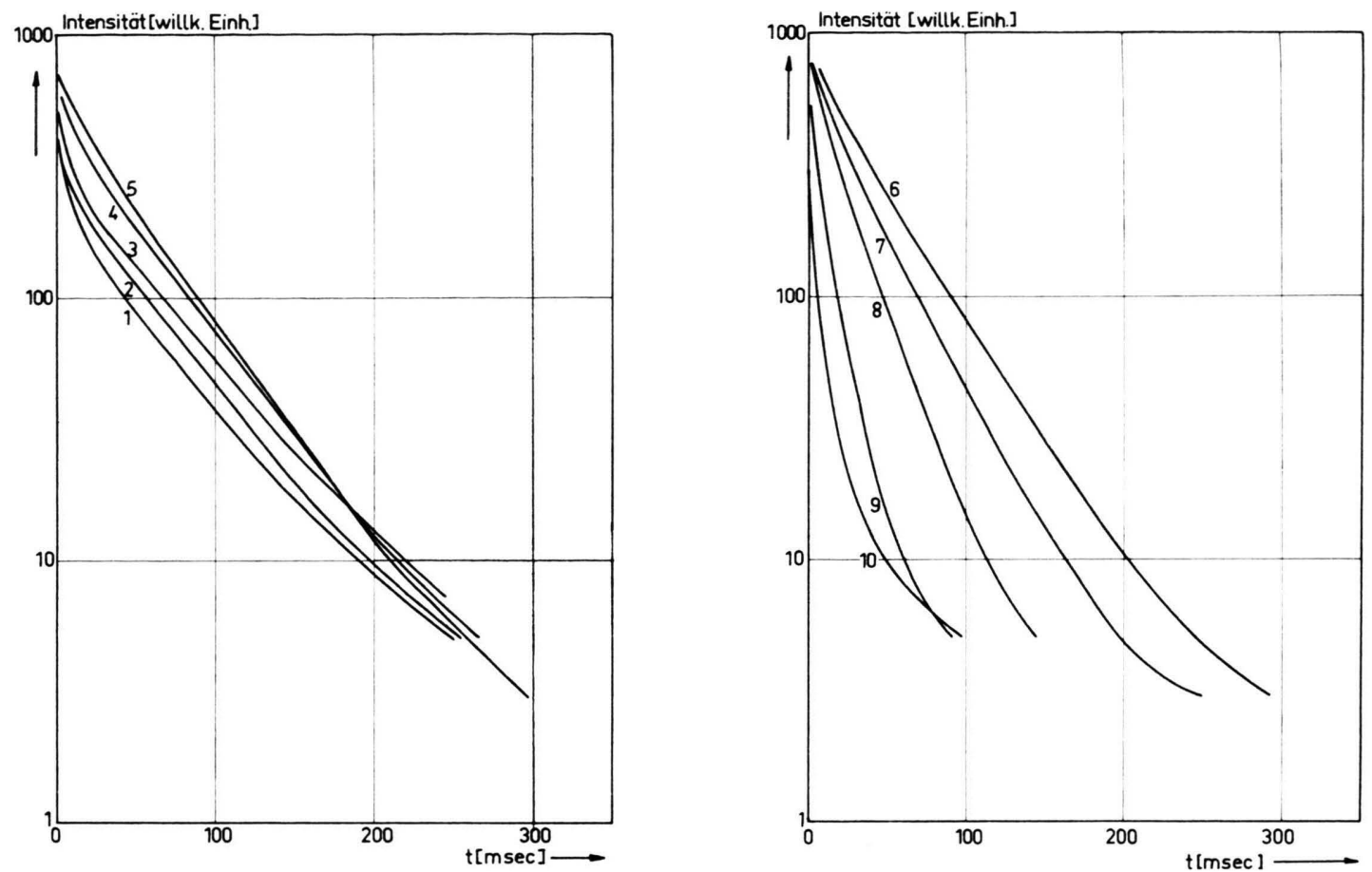

Abb. 6 a, b. Abklingkurven der Phosphoreszenz von Phenanthren $P_{30}$ bei verschiedenen Temperaturen. Anregungsdauer: 10 ms. (1) 77 , (2) 120 , (3) 150 , (4) 185 , (5) 220 , (6) 245 , (7) 270 , (8) 295 , (9) 330 , (10) $345^{\circ} \mathrm{K}$.

Phenanthren aber bei $21033 \mathrm{~cm}^{-1}$, also beträchtlich kürzer als die von uns beobachtete langwellige Nachleuchtkomponente, so daß es sich wahrscheinlich nicht um die Phenanthrenphosphoreszenz handelt. Durch vergleichende Messungen an Phenanthren mit Anthracenzusätzen wurde die Lage des 0,0-Übergangs der Anthracen $(\mathrm{T} \rightarrow \mathrm{S})$-Phosphoreszenz bei etwa $15000 \mathrm{~cm}^{-1}$ ermittelt (nach ${ }^{20,21}$ in EPA bei $14.928 \mathrm{~cm}^{-1}$ ). Es kommt also auch nicht die Anthracenphosphoreszenz in Betracht. Es muß sich um die Phosphoreszenz einer noch nicht identifizierten Verunreinigung handeln. Das zeitliche Abklingen der langwelligen Nachleuchtkomponente verläuft über einen Intensitätsbereich von mehr als einer Zehnerpotenz exponentiell (Abb.6a, b) und biegt dann in einen annähernd hyperbolischen Abfall ab (Abb. 7). Die Abklingkonstante des exponentiellen Anteils besitzt eine Temperaturabhängigkeit, die auf einen strahlungslosen Konkurrenzprozeß zur Emission schließen läßt mit einer Aktivierungsenergie von $0,28 \mathrm{eV}$ (Abb. 8). Der Temperaturverlauf der stationären Phosphoreszenzintensität stimmt mit dieser Vorstellung allerdings nicht überein.

20 S. P. McGlynn, M. R. Padhye u. M. Kasha, J. Chem. Phys. 23, 593 [1955].

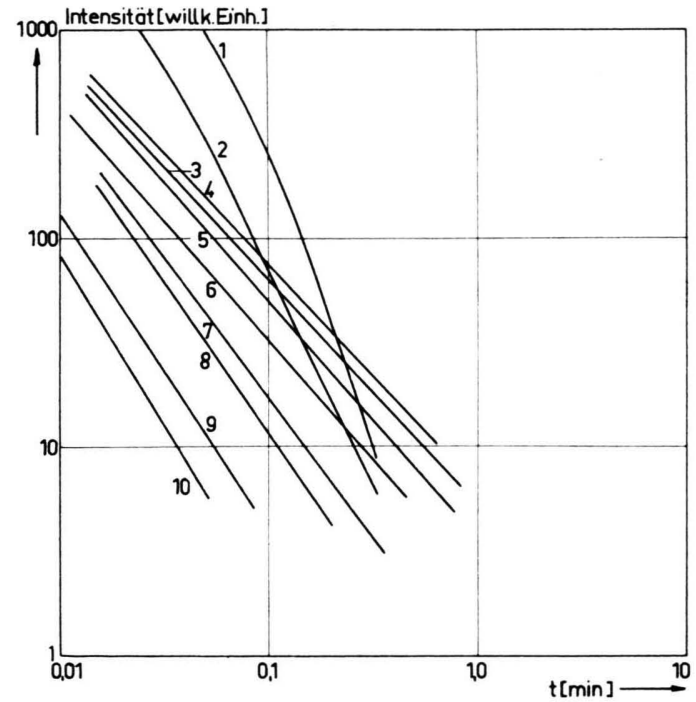

Abb. 7. Späteres Abklingen der Phosphoreszenz von Phenanthren $\mathrm{P}_{30}$ bei verschiedenen Temperaturen. Anregungsdauer: $1 \mathrm{~s}$. Temperaturen wie in Abb. 6.

21 S. P. McGlynn, J. D. Boggus u. E. Elder, J. Chem. Phys. 32, 357 [1960]. 


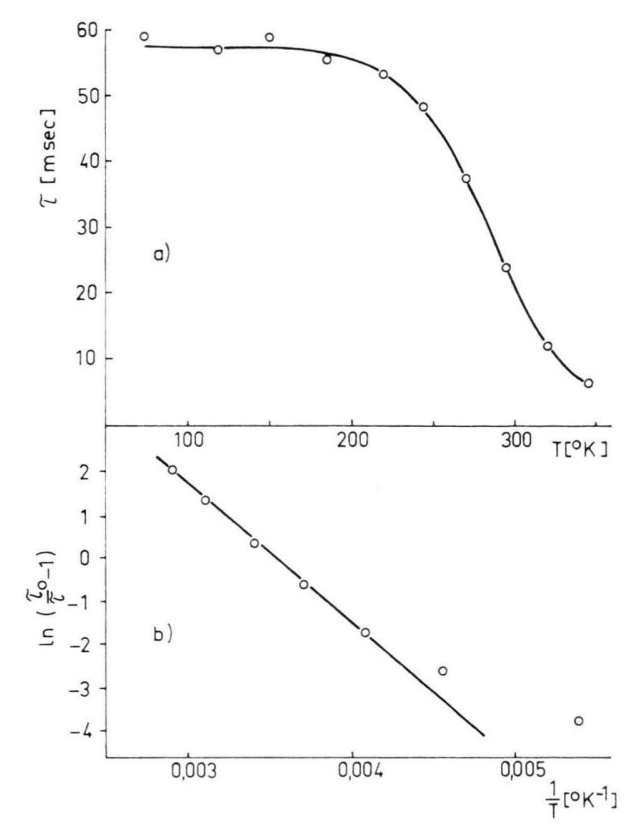

Abb. 8. a) Abklingdauern der exponentiellen Komponente der Phosphoreszenz in Abhängigkeit von der Temperatur (Probe $\mathrm{P}_{30}$ ). b) Aktivierungsgerade: $\Delta E=0,28 \mathrm{eV}$.

Der zeitliche Verlauf der kurzwelligen Komponente (verzögerte Fluoreszenz) ist weniger einfach zu beschreiben. In dem Temperaturbereich, in dem die verzögerte Fluoreszenz relativ intensiv auftritt, ist eine anfänglich exponentielle Abklingkomponente vorhanden. Die dazu gehörende Abklingzeit ist etwa halb so groß wie jene der Phosphoreszenz (Tab. 1). Diesem Anteil schließt sich ein hyperbolischer Abfall mit einem Exponenten $\alpha \approx 2$ an (Abb. 9). Die hyperbolische Komponente zeigt keine deutliche Temperaturabhängigkeit. Bei tieferen Temperaturen ist der Abklingvorgang von Anfang an hyperbolisch. Die Hyperbelexponenten des anfänglichen Verlaufs sind für verschiedene Temperaturen in Tab. 2 zusammengestellt. Der spätere Verlauf folgt auch hier wieder eher einer Hyperbel mit $\alpha \approx 2$.

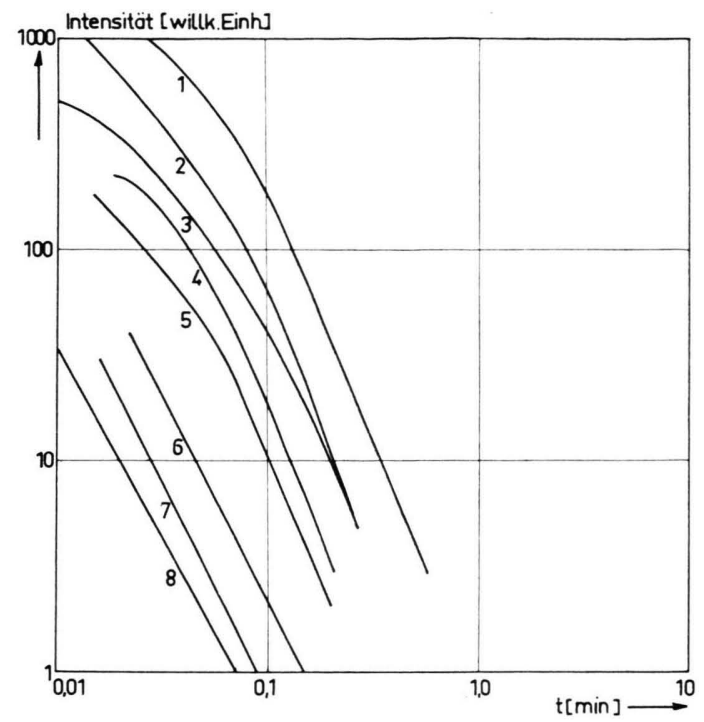

Abb. 9. Späteres Abklingen der verzögerten Fluoreszenz von Phenanthren $\mathrm{P}_{30}$ bei verschiedenen Temperaturen. Anregungsdauer: 1 s. (1) 77, (2) 120 , (3) 150 , (4) 185, (5) 220, (6) 245 , (7) 270, (8) $295^{\circ} \mathrm{K}$.

Bei starker ungefilterter Einstrahlung kann das Nachleuchten bis zu 15 Minuten lang verfolgt werden. Die langdauernde Energiespeicherung wurde mit Thermolumineszenzmessungen weiter untersucht.

Wurden die Proben bei $77^{\circ} \mathrm{K}$ angeregt und anschließend genügend rasch $\left(30-120^{\circ} \mathrm{K} / \mathrm{min}\right)$ aufgewärmt, so konnte eine deutliche Thermolumineszenz gemessen werden. Die Lichtsumme der thermisch stimulierten Emisison war bis zu Wartezeiten von 1 Stunde Dauer nach der Anregung bei $77^{\circ} \mathrm{K}$ bei gleichen Anregungsdauern bis auf 10\% konstant. Unter sonst konstanten Bedingungen ergab sich bei verschiedenen zeitlichen Temperaturgradienten (zwischen 30 und $120^{\circ} \mathrm{K} / \mathrm{min}$ ) eine bis auf ca. $10 \%$ konstante Lichtsumme. Bei kurzen Anregungsdauern nahm diese linear mit der Anregungsdauer zu.

Es wurde ohne Erfolg versucht, die gespeicherte Energie mit UR-Strahlung ,auszuleuchten“.

\begin{tabular}{|c|c|c|c|c|c|c|c|c|c|c|}
\hline$T\left({ }^{\circ} \mathrm{K}\right)$ & 80 & 120 & 150 & 185 & 220 & 245 & 270 & 295 & 330 & 345 \\
\hline$\tau_{\mathrm{P}}(\mathrm{ms})$ & 59 & 57 & 59 & 56 & 53,5 & 48,5 & 38 & 24 & 12 & $(6,5)$ \\
\hline$\tau_{\mathrm{F}}(\mathrm{ms})$ & - & - & - & - & - & $(23,5)$ & 17,5 & 13,0 & 7,0 & $3,5)$ \\
\hline
\end{tabular}

Tab. 1. Lebensdauern von Triplettphosphoreszenz $\tau_{\mathrm{P}}$ und verzögerter Fluoreszenz $\tau_{\mathrm{F}}$ bei verschiedenen Temperaturen (eingeklammerte Werte unsicher).

\begin{tabular}{|c|c|c|c|c|c|c|c|c|c|c|}
\hline$T\left({ }^{\circ} \mathrm{K}\right)$ & 80 & 120 & 150 & 185 & 220 & 245 & 270 & 295 & 330 & 345 \\
\hline$\alpha$ & 0,92 & 1,08 & 1,00 & 1,02 & 1,06 & $(1,06)$ & $(0,92)$ & - & $(0,92)$ & $(1,12)$ \\
\hline
\end{tabular}

Tab. 2. $\alpha$-Werte des Abklingens der verzögerten Fluoreszenz in Abhängigkeit von der Temperatur [Annahme: Abklingvorgang gemäß $I=I_{0}(a+b t)^{-\alpha}$; ( (eingeklammerte Werte unsicher). 
Aus Intensitätsgründen konnte die Thermolumineszenzstrahlung nur grob spektral zerlegt werden. Ihr Spektrum fällt mit den beiden Emissionsgebieten des Nachleuchtens zusammen. Man kann also annehmen, daß es sich in beiden Fällen um die gleichen Leuchtzentren und Elektronenübergänge handelt. Die kurz- und langwellige Komponente wird im folgenden als Thermofluoreszenz und Thermophosphoreszenz unterschieden.

Die Glow-Kurven von Thermofluoreszenz und -phosphoreszenz sind nur schlecht reproduzierbar aber doch deutlich voneinander verschieden. Für die Thermofluoreszenz erhielt man ein ausgeprägtes Maximum, aus dem man nach der Urbachschen Formel auf eine Aktivierungsenergie von ungefähr $0,3 \mathrm{eV}$ für die gespeicherte Anregungsenergie schließen kann. Die Thermophosphoreszenzkurve erstreckt sich von 100 bis über $300{ }^{\circ} \mathrm{K}$ ohne deutliches Maximum (Abb. 10).

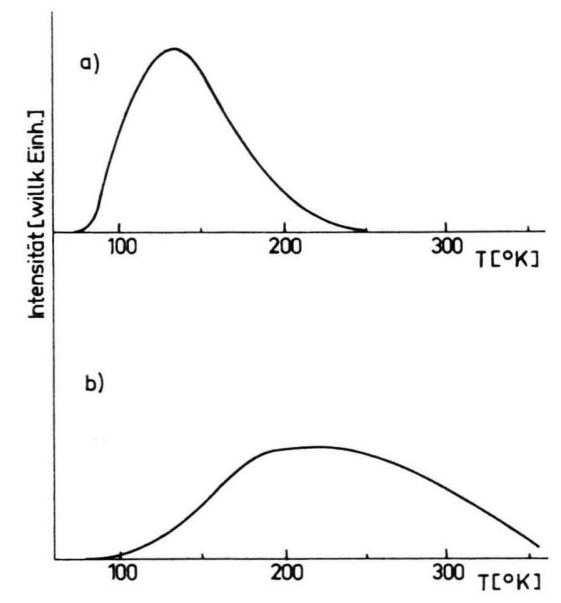

Abb. 10. Thermolumineszenzkurven von Phenanthren $\mathrm{P}_{30}$. Temperaturgradient: $80^{\circ} \mathrm{K} / \mathrm{min}$.

a) Thermofluoreszenz, b) Thermophosphoreszenz.

Überraschenderweise zeigten frische Phenanthrenproben nach langsamer Abkühlung von 350 auf $77{ }^{\circ} \mathrm{K}$ fast keine Thermolumineszenz. Erst nach 20 bis 30 Minuten dauernder UV-Bestrahlung bei Zimmertemperatur wurde die Probe thermolumineszenzfähig. Sie zeigte dann nur eine Thermophosphoreszenz - keine Thermofluoreszenz. Durch erneutes Tempern bei $350{ }^{\circ} \mathrm{K}$ läßt sich der ganze Vorgang wiederholen. Die Abkühlungsgeschwindigkeit hat auf die Thermophosphoreszenz keinen Einfluß. Sie ist aber entscheidend für das Auftreten einer Thermofluoreszenz. Während abgeschreckte Proben (von
350 auf $77^{\circ} \mathrm{K}$ ) eine ausgeprägte Fähigkeit zur Thermofluoreszenz besitzen, fehlt diese bis auf einen geringen Rest in langsam abgekühlten Proben.

\section{Diskussion}

Aus den Spektren ist zu entnehmen, daß bei den meisten unserer Proben die Fluoreszenz von Fremdmolekülen ausgeht. Doch deckt sich das bei $160{ }^{\circ} \mathrm{K}$ aufgenommene Spektrum einiger hochgereinigter Proben (z. B. P ${ }_{450}^{25}$, Abb. 2 c) bei einer Verschiebung um $300 \mathrm{~cm}^{-1} \mathrm{zu}$ höheren Wellenzahlen mit dem bei $77^{\circ} \mathrm{K}$ aufgenommenen Fluoreszenzspektrum von Phenanthren in Äthanol. (Die Wellenzahlen der charakteristischen Maxima stimmen aber nicht mit den von Benz und $\mathrm{WoLF}^{3}$ auf Grund ihrer Analyse angegegebenen Werten überein.)

Die Veränderungen im Fluoreszenzspektrum mit der Temperatur bei der Probe $\mathrm{P}_{450}^{25}$ deuten darauf hin, daß bei $77^{\circ} \mathrm{K}$ die Emission von längerwellig fluoreszierenden Zentren (X-Fallen nach ${ }^{3}$ ) ausgeht. Mit wachsender Temperatur tritt diese Emission zugunsten der kürzerwelligen eigentlichen Kristallemission des Phenanthrens zurück, so daß bei $160{ }^{\circ} \mathrm{K}$ im wesentlichen das Kristallspektrum des Phenanthrens emittiert wird.

Problematischer ist der Mechanismus der Energiespeicherung. Die besprochenen Experimente zeigen, $\mathrm{da}$ @ das Nachleuchten und die thermisch stimulierbare Emission aus zwei spektroskopisch unterscheidbaren Komponenten bestehen: einer verzögerten Kristall- oder Fehlstellenfluoreszenz und einer längerwelligen Triplettphosphoreszenz einer nicht bekannten Verunreinigung.

Die verzögerte Fluoreszenz enthält in dem Temperaturbereich, in dem sie besonders intensiv auftritt $\left(250-300{ }^{\circ} \mathrm{K}\right)$, einen anfänglich exponentiell abklingenden Teil, dessen Zeitkonstante etwa gleich der halben Zeitkonstanten der anfangs gleichfalls exponentiell verlaufenden Phosphoreszenz ist. Dieser Anteil dürfte auf der bekannten ${ }^{13},{ }^{16-18}$ verzögerten Fluoreszenz durch Vereinigung zweier Triplettenergien beruhen.

Die langsamer, nicht exponentiell abklingenden Ausläufer der verzögerten Fluoreszenz und der Phosphoreszenz können aber, wie der verschiedene zeitliche Verlauf und die unterschiedliche Temperaturabhängigket zeigen, nur aus zwei voneinander unabhängigen Energiespeichern versorgt werden. Die 
vorliegenden Ergebnise lassen nur vage Vermutungen über die Art dieser Speicher zu.

Das Termschema in Abb. 11 stellt einen noch sehr spekulativen Erklärungsversuch dar. Nach einer von SPONER ${ }^{8}$ vorgeschlagenen Möglichkeit werden neben

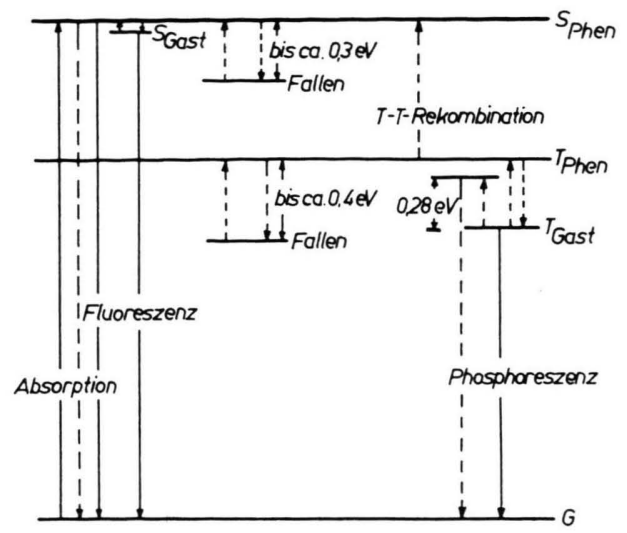

Abb. 11. Vorschlag eines Termschemas zur Beschreibung der Phenanthren-Kristallumineszenz. Erläuterung im Text.

dem heute allgemein angenommenen Singulett- und Triplett-Excitonenband lokalisierte „Excitonenhaftstellen" postuliert. Es müssen Stellen sein, an denen die Excitonenenergie zur Umwandlung eines Moleküls in eine stabile, aber reversible Modifikation höherer Energie verwandt wird. Unter Aufwendung einer Aktivierungsenergie kann die Rückkehr in den Normalzustand unter Freisetzung der Energie und Emission derselben erfolgen. Die bisherigen Messungen geben Anhaltspunkte über die Größenordnung der Aktivierungsenergien der beiden Haftstellensorten, der Singulett- und Triplett-Excitonenhaftstellen. Das Zeit- und Temperaturverhalten zwingt zur Annahme einer nicht näher bekannten Verteilung der Aktivierungsenergie in beiden Fällen (Argumes.ation wie in ${ }^{22}$ ) mit Maximalwerten von 0,3 bzw. 0,4 $\mathrm{eV}$ entsprechend der Abb. 11.

Da Thermofluoreszenz nur in abgeschreckten Kristallen stärker auftritt, Thermophosphoreszenz nur nach längerer UV-Bestrahlung, sind die Singuletthaftstellen möglicherweise an das Vorhandensein von Gitterfehlstellen gebunden, die Tripletthaftstellen dagegen mit photochemischen Umwandlungen in Zusammenhang zu bringen. Die Sättigung der Lichtsumme hängt vielleicht mit einer Erschöpfung eines Reaktionspartners (okkludiertes $\mathrm{O}_{2}$ ) zusammen.

Eine Bestätigung dieser Annahmen und eine Präzisierung des Bildes muß weiteren Untersuchungen vorbehalten bleiben.

Die Arbeit wurde am Physics Department der Duke University in Durham, North Caroline, USA, durchgeführt und von der National Science Foundation unterstützt. Frau Prof. H. Sponer-Franck danke ich für die Anregung und stete Förderung der Arbeit, Herrn Prof. W. Hanle für die Vermittlung und sein stetes Interesse. Dankbar bin ich auch für wertvolle Anregungen, die ich zu Beginn der Arbeit von Herrn Prof. J. Franck erhielt, sowie meinem verehrten Lehrer, Herrn Prof. A. Schmillen, für zahlreiche klärende Diskussionen und die Hilfe bei der Fertigstellung dieser Arbeit.

22 H. Kallmann, B. Kramer u. E. Sucov, J. Chem. Phys. 23, 1043 [1955]. 